\title{
Erratum: Electroweak form factors of heavy-light mesons: A relativistic point-form approach [Phys. Rev. D 86, 053010 (2012)]
}

\author{
María Gómez-Rocha and Wolfgang Schweiger
}

(Received 3 December 2020; published 22 December 2020)

DOI: 10.1103/PhysRevD.102.119901

We have discovered an error in part of the numerical results presented in the article.

The electromagnetic and pseudoscalar-to-pseudoscalar weak form factors have been calculated with the transpose of the Wigner $D$ matrices instead of with the Wigner $D$ matrices. Whereas the formulas in the paper are correct, there was a misprint in the code that produced wrong numerical results. The correction of this mistake produces a small change in Fig. 2 that, however, is irrelevant for the corresponding discussion.

It, however, is of relevance for Fig. 3 and the following discussion. The correct result shows that the physical form factor in the heavy-quark limit $f\left(\underline{v}_{\alpha} \cdot \underline{v}_{\alpha}^{\prime}, \nu_{\alpha}\right)$ does not change with $\nu_{\alpha}$. It is constant in the whole range and not only for large values of $\nu_{\alpha}$ (cf. figure below). Likewise, the spurious form factor $g\left(\underline{v}_{\alpha} \cdot \underline{v}_{\alpha}^{\prime}, \nu_{\alpha}\right)$ turns out to vanish completely in the heavy-quark limit, as it is shown in the correct Fig. 3. This finding lets us conclude that the effects of wrong cluster properties vanish in the heavy-quark limit in contrast to what has been stated originally. The heavy-quark limit removes the dependence of the form factors on the velocity $\nu_{\alpha}$ completely and suppresses the spurious form factor.
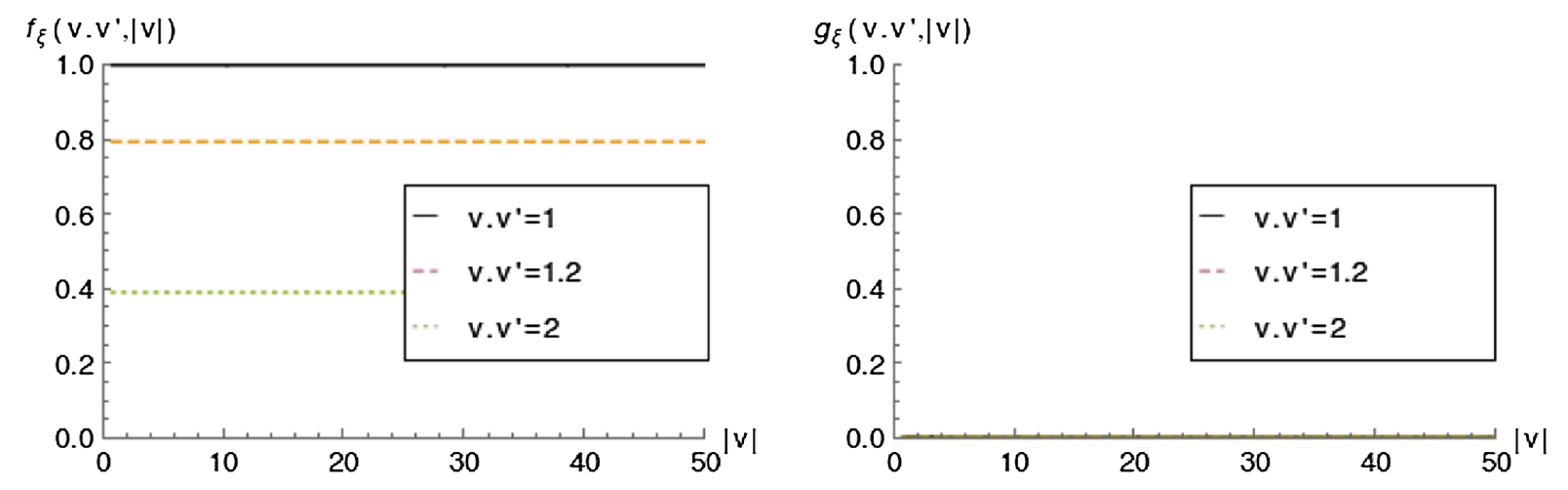

In the article, this affects the discussion in the paragraph below Eq. (54) [until Eq. (55)], as well as in the second paragraph of second column on page 18. Hardly visible changes occur also in Figs. 5 and 6 (left panel), i.e., figures for mesons of finite masses, but they are not relevant to the investigation treated in this article. The rest of the conclusions and comments in the article remain the same. For readers interested in more details of the numerics related to these transition form factors we refer to Ref. [1], where a larger number of processes has been calculated correctly.

[1] M. Gómez-Rocha, Phys. Rev. D 90, 076003 (2014). 